\title{
Vaccine-Induced Immune Thrombotic Thrombocytopenia (VITT): Targeting Pathomechanisms with Bruton Tyrosine Kinase Inhibitors
}

\author{
Philipp von Hundelshausen ${ }^{1,2}$ Reinhard Lorenz ${ }^{1}$ Wolfgang Siess ${ }^{1,2}$ Christian Weber $^{1,2,3,4, * \odot}$ \\ 1 Institute for Cardiovascular Prevention (IPEK), LMU Munich, Munich, Germany \\ ${ }^{2}$ DZHK (German Centre for Cardiovascular Research), partner site \\ Munich Heart Alliance, Munich, Germany \\ ${ }^{3}$ Department of Biochemistry, Cardiovascular Research Institute \\ Maastricht (CARIM), Maastricht University Medical Centre, \\ Maastricht, The Netherlands

\begin{abstract}
Address for correspondence Wolfgang Siess, Prof. Dr. med., Institut für Prophylaxe und Epidemiologie der Kreislaufkrankheiten, Klinikum Innenstadt, Ludwig-Maximilians Universität München, Pettenkoferstr. 9, D-80336 München, Germany

(e-mail: wsiess@med.uni-muenchen.de).
\end{abstract}

${ }^{4}$ Munich Cluster for Systems Neurology (SyNergy), Munich, Germany

Thromb Haemost 2021;121:1395-1399.

The review process for this paper was fully handled by Gregory Y. H. Lip, Editor-in-Chief.
A series of cases with rare thromboembolic incidents including cerebral sinus vein thrombosis (some of them fatal) and concomitant thrombocytopenia occurring shortly after vaccination with the coronavirus disease 2019 (COVID-19) vaccine AZD1222 (Vaxzevria) have caused significant concern and led to its temporary suspension in many countries. Immediate laboratory efforts in four of these patients have identified a tentative pathomechanism underlying this syndrome termed initially vaccine-induced prothrombotic immune thrombocytopenia (VIPIT) and renamed recently vaccineinduced immune thrombotic thrombocytopenia (VITT). It encompasses the presence of platelet-activating antibodies to platelet factor-4/heparin complexes, possibly emulated by polyanionic constituents of AZD1222, and thus resembles heparininduced thrombocytopenia (HIT). Because these immune complexes bind and activate platelets via Fcy receptor IIA (FcyRIIA), high-dose intravenous immunoglobulin G has been suggested for treatment of VITT in addition to non-heparin anticoagulants. Here we propose inhibitors of Bruton tyrosine kinase (Btk) approved for B cell malignancies (e.g., ibrutinib) as another therapeutic option in VITT, as they are expected to pleiotropically target multiple pathways downstream of FcyRIIA-mediated Btk activation, for example, as demonstrated for the effective inhibition of platelet aggregation, dense granule secretion, P-selectin expression and platelet-neutrophil aggregate formation stimulated by FcyRIIA cross-linking. Moreover, C-type lectin-like receptor CLEC-2- and GPIb-mediated platelet activation, the interactions and activation of monocytes and the release of neutrophil extracellular traps, as encountered in HIT, could be attenuated by Btk inhibitors. As a paradigm for emergency repurposing of approved drugs in COVID-19, off-label use of Btk inhibitors in a low-dose range not affecting haemostatic functions could thus be considered a sufficiently safe option to treat VITT. received

April 6, 2021

accepted

April 9, 2021

published online

April 13, 2021 (c) 2021. Thieme. All rights reserved. Georg Thieme Verlag KG,

Rüdigerstraße 14,

70469 Stuttgart, Germany
DOI https://doi.org/

10.1055/a-1481-3039.

ISSN 0340-6245. 
Vaccines are critical to effectively contain the coronavirus disease 2019 (COVID-19) pandemic. Four vaccines have been approved by the European Medicines Agency (EMA) as of 30 March 2021: two mRNA-based vaccines encoding the spike protein of severe acute respiratory syndrome coronavirus 2 (SARS-CoV-2) from Pfizer/BioNTech and Moderna, and two recombinant adenoviral vector-based vaccines encoding the spike protein from AstraZeneca/Oxford University and Janssen/Johnson\&Johnson. More than 120 million doses of vaccine had been administered in Europe (https:// ourworldindata.org/covid-vaccinations) by 31 March 2021). Despite unprecedented scientific and industrial efforts, supply of vaccines still falls short of urgent demand to reach protective immunity in the general population, increasing the pressure to use all approved vaccines.

More than 20 million doses of the COVID-19 vaccine AstraZeneca (AZD1222, ChAdOx1 nCoV-19) have been administered in European Union (EU) countries and the United Kingdom as of 25 March 2021. ${ }^{1}$ However, recent reports of rare severe cerebral venous sinus thrombosis (CVST) shortly after vaccination have prompted the temporary suspension of the vaccine in 16 continental European countries in midMarch. On 26 March 2021, EMA authorized the further use of the COVID-19 vaccine AstraZeneca, which was renamed Vaxzevria. ${ }^{2}$ According to the Robert Koch Institute, 2.7 million first doses and 767 second doses of the vaccine were given in Germany as of 29 March 2021, and a total of 31 cases of CVST in Germany after vaccination with Vaxzevria had been reported to the Paul-Ehrlich Institute as of 29 March 2021. Coincident thrombocytopenia was documented in 19 cases. In nine cases, the outcome was fatal. With the exception of two men, 36 and 57 years old, all reports concerned women aged 20 to 63 years. ${ }^{3}$ German authorities decided therefore on 30 March 2021 to suspend Vaxzevria for regular vaccinations of persons younger than 60 years. However, in the United Kingdom where 13.7 million doses of this vaccine have been applied, only 5 comparable cases have been reported so far in the media, ${ }^{4}$ initially hinting at batchspecific effects related to adenoviral vaccine constituents. More recently, a United Kingdom government update through 21 March 2021 documented 22 reports of CVST and 8 reports of other thrombosis events with low platelet counts. ${ }^{5}$ As of 4 April 2021, a total of 169 cases of CVST and 53 cases of splanchnic vein thrombosis were reported to EudraVigilance. ${ }^{6}$ Around 34 million people had been vaccinated with Vaxzevria in the European Economic Area (EEA) and United Kingdom by this date. On April 7, EMA's safety committee (Pharmacovigilance Risk Assessment Committee [PRAC]) had concluded that unusual blood clots with low blood platelet counts should be listed as very rare side effects of Vaxzevria. ${ }^{6}$

A cooperative effort led by Andreas Greinacher at Greifswald University rapidly unravelled a tentative pathogenic mechanism underlying these rare incidents of mostly intracranial venous thromboses associated with thrombocytopenia, which they named vaccine-induced prothrombotic immune thrombocytopenia (VIPIT) and that was renamed recently vaccine-induced immune thrombotic thrombocy- topenia (VITT) (see section "Note"). ${ }^{7}$ They were alarmed by an index patient (female) with a splanchnic, followed by a cerebral venous and aortal thrombosis shortly after the first vaccination, and documented a series of eight similar cases (seven females and one male), seven presenting cerebral vein thrombosis and one with pulmonary embolism. All had

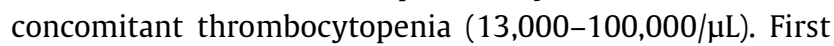
symptoms had been observed from 4 to 16 days after vaccination, and four patients died. Sera of four patients were available for further investigations. In all four patients, antibodies directed against platelet factor-4 (PF4)/heparin complexes were found and these sera activated washed test platelets from normal donors weakly in the absence and strongly in the presence of added PF4. Thus, laboratory findings in these rare incidents after Vaxzevria vaccination resembled heparin-induced thrombocytopenia (HIT), a thrombocytopenic prothrombotic disorder caused by the formation of immunoglobulin $\mathrm{G}$ ( $\operatorname{IgG}$ ) antibodies against new epitopes exposed after association of heparin or other polyanions with PF4 (CXCL4) secreted from platelets. ${ }^{8}$ By their Fc domains, these immune complexes bind to FcrRIIA on the surface of platelets and thus cross-link these receptors and induce platelet activation. ${ }^{8,9}$ Indeed platelet activation by the VITT sera was inhibited by high concentrations of either heparin or IgG shielding FcrRIIA. ${ }^{7}$ Interestingly, direct addition of the AZD1222 vaccine to washed platelets or first pre-incubating the platelets with diluted vaccine and subsequently washing them enhanced platelet reactivity to VITT sera in the presence of PF4. In analogy to heparin, polyanionic deoxyribonucleic acid (DNA) or after cleavage by deoxyribonuclease (DNase), polyanionic DNA fragments or nucleoprotein of AZD1222 might pre-activate platelets, as well as spike protein, if transcribed in excess and binding to angiotensin-converting enzyme 2 (ACE2) on platelets as has been suggested for Sars-Cov-2. ${ }^{10}$ Until vaccine virus polymerase chain reaction (PCR) data might become available, it can only be speculated whether this can occur in vivo under rare circumstances, for example, during coincident infection with a wild-type virus substituting cross-functional E1 gene that is deleted in the vaccinia adenovirus to abrogate its replication. ${ }^{11} \mathrm{~A}$ sensitization of platelets may likewise occur during other forms of coincidental infection or superinfection. Taken together, this possibility would support a concept implicating polyanionic components of AZD1222 as binding partners for PF4 causing the prothrombotic disorder. Based on these striking analogies with HIT, the authors suggested non-heparin anticoagulants and high-dose intravenous immunoglobulin G (IVIG) to treat VITT. ${ }^{7}$

As IVIG might not be available globally for pathogenesisguided experimental therapy of VITT, we want to draw attention to an additional pharmacologic option that could block fatal platelet activation by the FcrRIIA pathway apparently operative in VITT: platelet FcrRIIA stimulation leads to downstream activation of Bruton tyrosine kinase (Btk) $)^{12}$ as a decisive signalling pathway for subsequent steps of platelet activation. ${ }^{13}$ We have recently shown that platelet activation (including aggregation, dense granule secretion and $P$-selectin expression) and formation of platelet-neutrophil 
aggregates stimulated by FcrRIIA cross-linking or sera from HIT patients were completely suppressed by incubating blood with low concentrations of several Btk inhibitors (BTKi) in vitro. ${ }^{13}$ Approved BTKi are now widely used as standard drugs for the long-term oral therapy of several B cell malignancies with a remarkable safety profile ${ }^{14}$ and exerted an apparently protective effect in case of coincident symptomatic COVID-19. ${ }^{15,16}$ Of note, the platelet-inhibitory concentrations of the approved BTKi ibrutinib, acalabrutinib, zanubrutinib and tirabrutinib in blood were much lower than the drug levels reached in patients treated with oral standard doses for B cell disorders. ${ }^{13,17}$ Furthermore, intake of a single dose of ibrutinib $(280 \mathrm{mg}$ ) by human healthy volunteers rapidly blocked ( 3 hours after intake) platelet aggregation and secretion on maximal stimulation of FcrRIIA on platelets in blood ex vivo. ${ }^{13}$ Stimulus/receptorselective platelet inhibition was sustained for up to 2 days, which is explained by covalent binding of ibrutinib to Btk and the lack of de novo protein synthesis in platelets. Suppression of Btk-mediated platelet activation has previously been shown to be maintained by low ibrutinib dosage $(140 \mathrm{mg}$ per day or on alternate days). ${ }^{18,19}$

The pathogenesis of HIT and obviously likewise VITT involves other cells in addition to platelets including monocytes, neutrophils and endothelial cells. BTKi suppressed Pselectin expression on platelets, ${ }^{13}$ which is crucially involved in their interaction with monocytes to promote tissue factor expression amplifying thrombin formation. ${ }^{20,21}$ BTKi also inhibit FcrRIIA-mediated stimulation of monocytes and neutrophils. ${ }^{22}$ Neutrophil accumulation and neutrophil extracellular trap (NET) release contribute to thrombosis in $\mathrm{HIT}^{23}$ and HIT immune complexes induce NET release via interaction with FcrRIIA on neutrophils and through neutrophil-platelet association ${ }^{24}$ inhibited by BTKi. ${ }^{13}$ Moreover, inhibition of autoreactive B-lymphocytes by BTKi is expected to reduce the production of pathogenic anti-PF4 antibodies. ${ }^{25}$ Overall, these findings establish a plethora of deleterious mechanisms beyond platelet activation that could be pleiotropically targeted by BTKi in the pathogenesis of VITT (-Fig. 1).

Whereas in COVID-19 signs of a general prothrombotic disposition are well documented, ${ }^{26,27}$ the uncommon predominant localization of thrombosis in cerebral sinus veins in VITT is puzzling. An additional localizing factor appears to be operative. In SARS-CoV-2 infection, neuro-invasion may occur and spike protein is involved, ${ }^{28}$ but CVST has not emerged as a prominent subset in the heterogeneous neurologic manifestations. ${ }^{29}$ From fatal cases of VITT, autopsybased evidence might become available to clarify if and by which mechanisms localized damage to the endothelium of cerebral sinus and splanchnic veins has occurred. Damaged endothelium could then bind circulating free and/or plateletbound PF4-IgG complexes in VITT-prone patients. PF4 has recently been shown to bind at multiple sites along the surface of extended strings of von Willebrand factor (VWF) released from the endothelium following photochemical injury. ${ }^{30}$ The PF4/VWF complexes were recognized by antibodies from HIT patients and promoted platelet adhesion

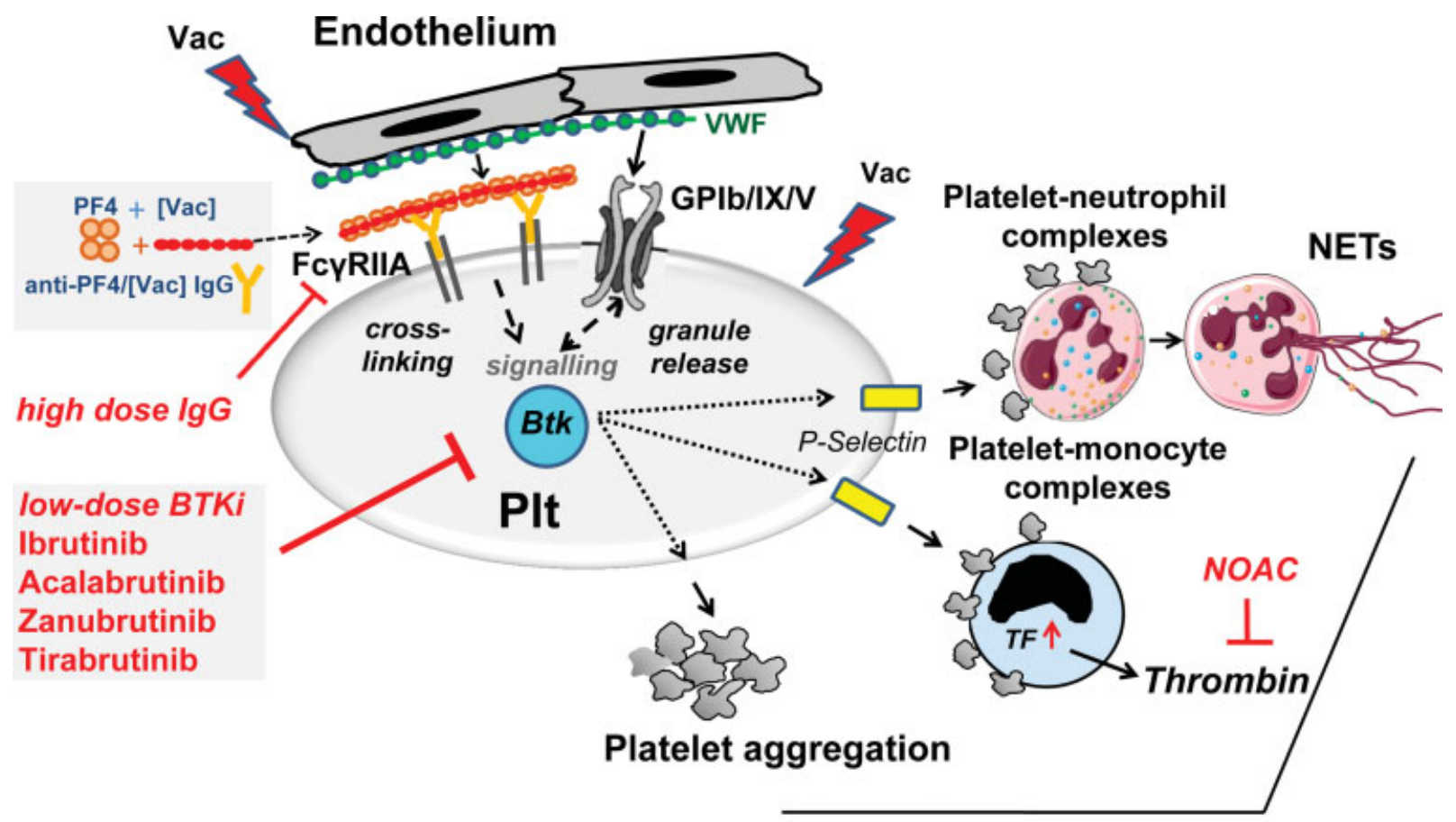

\section{Thrombotic occlusion}

Fig. 1 Model for the multiple roles of Bruton tyrosine kinase in the pathomechanisms of vaccine-induced immune thrombotic thrombocytopenia (VITT) and proposed therapeutic interventions. Btk, Bruton tyrosine kinase; BTKi, Bruton tyrosine kinase inhibitors; FcyRIIA, Fcy fragment of immunoglobulin (IgG) low-affinity IIA receptor; GPIb-V-IX, glycoproteins Ib, V, IX; NETs, neutrophil extracellular traps; NOAC, new oral anticoagulants; PF4, platelet factor-4; Plt, platelet; TF, tissue factor; VWF, von Willebrand factor; Vac, Vaxzevria; [Vac], polyanionic constituents of Vaxzevria. 
and thrombus growth under flow. Notably, platelet adhesion to the PF4-VWF-HIT antibody complexes was inhibited by antibodies that blocked not only FcrRIIA but also the GPIb-IX complex on platelets. Intriguingly, VWF stimulation of GPIb also activates Btk, ${ }^{31}$ and BTKi reduce VWF-/GPIb-mediated platelet activation in blood (as measured by ristocetininduced aggregation) and platelet adhesion to VWF surfaces under flow in vitro and ex vivo. ${ }^{13,18,19,32-34}$ Furthermore, Btk is critical in mediating platelet C-type lectin-like receptor 2 (CLEC-2) activation by podoplanin. ${ }^{35}$ Podoplanin is highly expressed in human thrombosed veins, ${ }^{36}$ mice with a deficiency in CLEC-2 are protected against deep vein thrombosis, ${ }^{37}$ and CLEC- 2 activation by podoplanin contributes to inflammation-driven murine hepatic thrombosis. ${ }^{38}$ Recently, low concentrations of ibrutinib and acalabrutinib have been shown to inhibit CLEC-2-stimulated platelet aggregation. $^{39}$ Taken together, this suggests additional possibly favourable effects of BTKi on VITT beyond inhibition of FcrRIIA on platelets.

As an alternative strategy, an inhibitor of spleen tyrosine kinase (Syk), which is also activated downstream of platelet FcrRIIA, ${ }^{40}$ could be considered. R406, the active metabolite of the Syk inhibitor fostamatinib has been shown to inhibit FcrRIIA-induced platelet activation triggered by heparin-PF4 autoantibodies in vitro, ${ }^{41}$ and fostamatinib has been recently approved for the treatment of therapy-resistant immune thrombocytopenia (ITP), an autoimmune disease characterized by autoantibodies against platelet GPIb/GPIX and $\alpha$ IIb $\beta 3$ integrin and classically treated with IVIG. ${ }^{42,43}$ By inhibiting Fc receptor signalling on macrophages and neutrophils that recognize platelet-bound antibodies, fostamatinib reduces platelet phagocytosis and removal, mainly in the spleen, ${ }^{33}$ and reverses thrombocytopenia. However, the therapeutic levels of R406 are apparently too low to directly inhibit platelets ex vivo. ${ }^{44}$ Similar observations have been made recently with the new Syk inhibitor entospletinib, ${ }^{45}$ at present in phase 3 clinical trials of acute myeloid leukaemia (AML).

In conclusion, several mechanisms amenable to inhibition by approved BTKi are at the centre of HIT and VITT pathophysiology according to the first key observations by Greinacher et al. ${ }^{7}$ In addition to ibrutinib, other irreversible BTKi have meanwhile been approved (acalabrutinib, zanubrutinib, tirabrutinib) for the treatment of B cell malignancies. ${ }^{46-49}$ Especially with concomitant thrombocytopenia, bleeding risks must be taken into account that might, however, be rather moderate and tolerable: the reversible covalent BTKi rilzabrutinib is currently being tested in clinical trials of ITP patients at doses, which inhibit Btk-mediated platelet activation in blood in vitro (von Hundelshausen et al, unpublished findings), and no bleeding events were reported after completing phase 2 , although at study entry patients had only a median platelet count of $\sim 14,000 / \mu \mathrm{L}$ (for reference, please see von Hundelshausen and Siess ${ }^{17}$ ). A shortterm application of approved BTKi in a low Btk-specific dosage that leaves other pathways of haemostatic platelet functions intact ${ }^{17-19,32}$ might be regarded as safe enough for a pathophysiology-guided compassionate off-label use in selected cases of VITT. Although the database is yet limited, this might represent a reasonable paradigm for emergency repurposing of approved drugs in COVID-19.

\section{Note}

After acceptance of this review, two articles have been published which describe further cases of vaccine-induced thrombotic thrombocytopenia (VITT). See below:

1. Greinacher A, Thiele T, Warkentin TE, et al. Thrombotic thrombocytopenia after ChadOx1 $\mathrm{nCoV}-19$ vaccination. N Engl J Med 2021;384(22):2092-2101

2. Schultz NH, Sørvoll IH, Michelsen AE, et al. Thrombosis and thrombocytopenia after ChadOx1 nCoV-19 vaccination. N Engl J Med 2021;384(22):2124-2130

\section{Funding}

This work was supported by the Deutsche Forschungsgemeinschaft (SFB1123-A1/A2/A10).

Conflict of Interest

None declared.

\section{Acknowledgements}

C.W. is a Van de Laar professor of atherosclerosis at CARIM, Maastricht University.

\section{References}

1 Mallapaty S, Callaway E. What scientists do and don't know about the Oxford-AstraZeneca COVID vaccine. Nature 2021;592 (7852):15-17

2 European Medicines Agency. Vaxzevria1 (Covid-19 vaccine (ChAdOx1-s [recombinant])). 2021. Report No.: EMA/182334/2021, EMEA/H/C/005675. Available at: https://www.ema.europa.eu/en/ documents/overview/vaxzevria-previously-covid-19-vaccineastrazeneca-epar-medicine-overview_en.pdf

3 Paul-Ehrlich-Institut. Sehr seltene Fälle von Hirnvenenthrombosen nach Impfung. Paul Ehrlich Institut; 2021. Available at: https:// www.pei.de/DE/service/presse/aktuelles/aktuelles-inhalt.html; jsessionid=7C63F1E4F9A2A12E296611B36952A6F0.intranet2414

4 ARD. Hohe Impfquote: Großbritannien setzt weiter auf Astrazeneca. 2021. Available at: https://www.ardmediathek.de/video/hohe-impf quote-grossbritannien-setzt-weiter-auf-astrazeneca/tagesschau24 /Y3JpZDovL2Rhc2Vyc3RILmRIL3RhZ2Vzc2NoYXUyNC85M-

TE3NDhlZC04NWU2LTRIMzUtODIxMy02NDI4ZGVIOWQ4ZDYvMQ/

5 UK.gov. Coronavirus vaccine: weekly summary of yellow card reporting. 2021. Available at: https://www.gov.uk/government/publications/coronavirus-covid-19-vaccine-adverse-reactions/coronavirusvaccine-summary-of-yellow-card-reporting

6 European Medicines Agency. EMA confirms overall benefit-risk remains positive. News. 2021. Available at: https://www.ema. europa.eu/en/news/astrazenecas-covid-19-vaccine-ema-findspossible-link-very-rare-cases-unusual-blood-clots-low-blood

7 Greinacher A, Thiele T, Warkentin TE, Weisser K, Kyrle P, Eichinger S. A prothrombotic thrombocytopenic disorder resembling heparin-induced thrombocytopenia following coronavirus-19 vaccination. Research Square 2021:1-8. Available at: https://doi.org/ 10.21203/rs.3.rs-362354/v1

8 Greinacher A. Clinical practice. Heparin-induced thrombocytopenia. N Engl J Med 2015;373(03):252-261

9 Arepally GM. Heparin-induced thrombocytopenia. Blood 2017; 129(21):2864-2872 
10 Zhang S, Liu Y, Wang X, et al. SARS-CoV-2 binds platelet ACE2 to enhance thrombosis in COVID-19. J Hematol Oncol 2020;13(01): 120

11 Almuqrin A, Davidson AD, Williamson MK, et al. SARS-CoV-2 vaccine ChAdOx1 nCoV-19 infection of human cell lines reveals low levels of viral backbone gene transcription alongside very high levels of SARS-CoV-2S glycoprotein gene transcription. Genome Med 2021;13(01):43

12 Oda A, Ikeda Y, Ochs HD, et al. Rapid tyrosine phosphorylation and activation of Bruton tyrosine/Tec kinases in platelets induced by collagen binding or CD32 cross-linking. Blood 2000;95(05): 1663-1670

13 Goldmann L, Duan R, Kragh T, et al. Oral Bruton tyrosine kinase inhibitors block activation of the platelet Fc receptor CD32a (FcrRIIA): a new option in HIT? Blood Adv 2019;3(23):4021-4033

14 Bond DA, Woyach JA. Targeting BTK in CLL: beyond ibrutinib. Curr Hematol Malig Rep 2019;14(03):197-205

15 Treon SP, Castillo JJ, Skarbnik AP, et al. The BTK inhibitor ibrutinib may protect against pulmonary injury in COVID-19-infected patients. Blood 2020;135(21):1912-1915

16 Scarfò L, Chatzikonstantinou T, Rigolin GM, et al. COVID-19 severity and mortality in patients with chronic lymphocytic leukemia: a joint study by ERIC, the European Research Initiative on CLL, and CLL Campus. Leukemia 2020;34(09):2354-2363

17 von Hundelshausen P, Siess W. Bleeding by Bruton tyrosine kinase-inhibitors: dependency on drug type and disease. Cancers (Basel) 2021;13(05):1103

18 Busygina K, Jamasbi J, Seiler T, et al. Oral Bruton tyrosine kinase inhibitors selectively block atherosclerotic plaque-triggered thrombus formation in humans. Blood 2018;131(24):2605-2616

19 Busygina K, Denzinger V, Bernlochner I, Weber C, Lorenz R, Siess W. Btk inhibitors as first oral atherothrombosis- selective antiplatelet drugs? Thromb Haemost 2019;119(08):1212-1221

20 Tutwiler V, Madeeva D, Ahn HS, et al. Platelet transactivation by monocytes promotes thrombosis in heparin-induced thrombocytopenia. Blood 2016;127(04):464-472

21 Thomas MR, Storey RF. The role of platelets in inflammation. Thromb Haemost 2015;114(03):449-458

22 Chang BY, Huang MM, Francesco M, et al. The Bruton tyrosine kinase inhibitor PCI-32765 ameliorates autoimmune arthritis by inhibition of multiple effector cells. Arthritis Res Ther 2011;13 (04):R115

23 Gollomp K, Kim M, Johnston I, et al. Neutrophil accumulation and NET release contribute to thrombosis in HIT. JCI Insight 2018;3 (18):99445

24 Perdomo J, Leung HHL, Ahmadi Z, et al. Neutrophil activation and NETosis are the major drivers of thrombosis in heparin-induced thrombocytopenia. Nat Commun 2019;10(01):1322

25 Pal Singh S, Dammeijer F, Hendriks RW. Role of Bruton tyrosine kinase in B cells and malignancies. Mol Cancer 2018;17(01):57

26 Bikdeli B, Madhavan MV, Jimenez D, et al; Global COVID-19 Thrombosis Collaborative Group, Endorsed by the ISTH, NATF, ESVM, and the IUA, Supported by the ESC Working Group on Pulmonary Circulation and Right Ventricular Function. Covid-19 and thrombotic or thromboembolic disease: implications for prevention, antithrombotic therapy, and follow-up-JACC stateof-the-art review. J Am Coll Cardiol 2020;75(23):2950-2973

27 McFadyen JD, Stevens H, Peter K. The emerging threat of (micro) thrombosis in Covid-19 and its therapeutic implications. Circ Res 2020;127(04):571-587

28 Aghagoli G, Gallo Marin B, Katchur NJ, Chaves-Sell F, Asaad WF, Murphy SA. Neurological involvement in Covid-19 and potential mechanisms: a review. Neurocrit Care 2020. Doi: 10.1007/ s12028-020-01049-4
29 Yachou Y, El Idrissi A, Belapasov V, Ait Benali S. Neuroinvasion, neurotropic, and neuroinflammatory events of SARS-CoV-2: understanding the neurological manifestations in COVID-19 patients. Neurol Sci 2020;41(10):2657-2669

30 Johnston I, Sarkar A, Hayes V, et al. Recognition of PF4-VWF complexes by heparin-induced thrombocytopenia antibodies contributes to thrombus propagation. Blood 2020;135(15): 1270-1280

31 Liu J, Fitzgerald ME, Berndt MC, Jackson CW, Gartner TK. Bruton tyrosine kinase is essential for botrocetin/VWF-induced signaling and GPIb-dependent thrombus formation in vivo. Blood 2006;108 (08):2596-2603

32 Denzinger V, Busygina K, Jamasbi J, et al. Optimizing platelet GPVI inhibition versus hemostatic impairment by ibrutinib and the novel Btk-inhibitors acalabrutinib, ONO/GS-4059, BGB-3111 and evobrutinib. Thromb Haemost 2019;119:397-406

33 Kazianka L, Drucker C, Skrabs C, et al. Ristocetin-induced platelet aggregation for monitoring of bleeding tendency in CLL treated with ibrutinib. Leukemia 2017;31(05):1117-1122

34 Levade M, David E, Garcia C, et al. Ibrutinib treatment affects collagen and von Willebrand factor-dependent platelet functions. Blood 2014;124(26):3991-3995

35 Rayes J, Watson SP, Nieswandt B. Functional significance of the platelet immune receptors GPVI and CLEC-2. J Clin Invest 2019; 129(01):12-23

36 Nicolson PL, Welsh JD, Chauhan A, Thomas MR, Kahn ML, Watson SP. A rationale for blocking thromboinflammation in COVID-19 with Btk inhibitors. Platelets 2020;31(05):685-690

37 Payne H, Ponomaryov T, Watson SP, Brill A. Mice with a deficiency in CLEC-2 are protected against deep vein thrombosis. Blood 2017;129(14):2013-2020

38 Hitchcock JR, Cook CN, Bobat S, et al. Inflammation drives thrombosis after Salmonella infection via CLEC-2 on platelets. J Clin Invest 2015;125(12):4429-4446

39 Nicolson PLR, Nock SH, Hinds J, et al. Low-dose Btk inhibitors selectively block platelet activation by CLEC-2. Haematologica 2021;106(01):208-219

40 Arman M, Krauel K. Human platelet IgG Fc receptor FcrRIIA in immunity and thrombosis. J Thromb Haemost 2015;13(06):893-908

41 Lhermusier T, van Rottem J, Garcia C, et al. The Syk-kinase inhibitor R406 impairs platelet activation and monocyte tissue factor expression triggered by heparin-PF4 complex directed antibodies. J Thromb Haemost 2011;9(10):2067-2076

42 Zufferey A, Kapur R, Semple JW. Pathogenesis and therapeutic mechanisms in immune thrombocytopenia (ITP). J Clin Med 2017;6(02):6

43 Miltiadous $\mathrm{O}$, Hou M, Bussel JB. Identifying and treating refractory ITP: difficulty in diagnosis and role of combination treatment. Blood 2020;135(07):472-490

44 Braselmann S, Taylor V, Zhao H, et al. R406, an orally available spleen tyrosine kinase inhibitor blocks fc receptor signaling and reduces immune complex-mediated inflammation. J Pharmacol Exp Ther 2006;319(03):998-1008

45 Series J, Ribes A, Garcia C, et al. Effects of novel Btk and Syk inhibitors on platelet functions alone and in combination in vitro and in vivo. J Thromb Haemost 2020;18(12):3336-3351

46 Imbruvica. Highlights of prescribing information. 2020. Available at: https://imbruvica.com/files/prescribing-information.pdf

47 Calquence. Highlights of prescribing information. 2020. Available at: https://www.azpicentral.com/calquence/calquence.pdf

48 Brukinsa. Highlights of Prescribing Information. 2020. Available at: https://www.brukinsa.com/prescribing-information.pdf

49 PMDA Japan. Velexbru (tirabrutinib). Japanese prescribing information. 2020. Available at: https://www.pmda.go.jp/files/000237315.pdf 